\title{
EFICÁCIA E SELETIVIDADE DO HERBICIDA 2,4-D, APLICADO EM DIFERENTES DOSES, ÉPOCAS E FORMULAÇÕES EM ARROZ IRRIGADO
}

\author{
José Alberto Noldin ${ }^{1}$ \\ 'Engº. Agrônomo, Ph.D., Pesquisador. Epagri - Estação Experimental de Itajaí. Caixa Postal 277. Itajaí, SC 88301-970 noldin@epagri.rct- \\ $\underline{\text { sc.br }}$
}

\begin{abstract}
RESUMO
Um experimento foi conduzido em campo, em duas estações de crescimento, na Estação Experimental de Itajaí da Epagri/SC (2656'35'S e 4845'37'W), nas safras 1996/97 e 1997/98, para avaliar a eficácia e seletividade de quatro doses, duas épocas de aplicação e duas formulações do herbicida 2,4-D em arroz irrigado, cultivar Epagri 108, no sistema prégerminado. As duas épocas de aplicação foram em pós-emergência normal, arroz e plantas daninhas com 3-4 folhas, e pósemergência tardia, 38-39 dias após a semeadura do arroz. As doses avaliadas foram: 2,4-D amina a 200, 400,800 e $1200 \mathrm{~g} /$ ha e.a. (equivalente ácido) e 2,4-D éster a 56, 100, 112, 200, 224, 336 e $600 \mathrm{~g} / \mathrm{ha}$ e.a. Geralmente, as doses mais elevadas de 2,4-D resultaram em sintomas de fitotoxicidade no arroz, em ambas as épocas de aplicação, mas variável entre safras. No entanto, a fitotoxicidade observada não afetou significativamente a esterilidade de espiguetas, pois as aplicações tardias foram efetuadas 20 a 30 dias antes do início do desenvolvimento das panículas do arroz. De maneira geral, observou-se que tanto a aplicação de 2,4-D éster como 2,4-D amina, aplicados nas doses de $100 \mathrm{~g} / \mathrm{ha}$ e.a. da formulação éster e $200 \mathrm{~g} / \mathrm{ha}$ e.a. da formulação amina, em pós-emergência normal, proporcionaram controle adequado de Fimbristylis miliacea, Ludwigia longifolia e L. octovalvis. A dose de 2,4-D necessária para o controle adequado de Heteranthera reniformis foi superior à requerida para as demais espécies presentes no experimento. Os resultados obtidos sugerem que a aplicação de 2,4-D, nas formulações amina ou éster, deve ser preferencialmente efetuada antes do perfilhamento do arroz e que sob tais condições podem ser utilizadas doses mais baixas com melhor controle das plantas daninhas e menor fitotoxicidade ao arroz.
\end{abstract}

Palavras-chave: 2,4-D amina, 2,4-D éster, fitotoxicidade, planta daninha.

\author{
ABSTRACT \\ Efficacy and selectivity of 2,4-D applied at different rates, growth stages and \\ formulations in paddy rice
}

An experiment was carried out at Epagri, in Itajaí, Santa Catarina State (2656’35'S and 4845'37'W), during 1996/ 97 and 1997/98 growing seasons, to evaluate the efficacy and selectivity of 2,4-D herbicide at four rates, two times of application and two formulations, in infested rice. Herbicides were applied postemergence, with rice plants at the 3-4 leaf stage, and late postemergence, 38-39 days after planting. Herbicide formulations and rates evaluated were: 2,4-D amine at 200, 400, 800 and $1200 \mathrm{~g} / \mathrm{ha}$ a.e. (acid equivalent) and ester at 56, 112, 224 and $336 \mathrm{~g} / \mathrm{ha}$ a.e. Overall results showed that higher 2,4-D rates of both formulations at both application timings, injured rice. However, crop injury did not increase spikelet sterility because the rice cultivar was a late season variety and the second application was carried out at least 30 days before panicle differentiation. Herbicide 2,4-D, ester or amine, applied postemergence, at low rates (100 g/ha a.e. of ester formulation or $200 \mathrm{~g} / \mathrm{ha}$ a.e. of amine formulation), controlled Fimbristylis miliacea, Ludwigia longifolia, and L. octovalvis. Heteranthera reniformis was less sensitive to 2,4-D, and higher rates were required for good control. These results suggest 


\section{José Alberto Noldin}

that 2,4-D application in rice should be carried out before tillering because, in such conditions, even low rates of the herbicide would provide good broadleaf and sedge control, besides minimizing crop injury.

Key words: 2,4-D amine, 2,4-D ester, rice injury, weed.

\section{INTRODUÇÃO}

O herbicida 2,4-D, um dos primeiros compostos desenvolvido para uso comercial no mundo, foi registrado nos Estados Unidos no ano de 1945 e desde a sua introdução no mercado, tem sido largamente utilizado (Burnside et al., 1996). Apesar de estar no mercado há mais de 50 anos, ainda constitui-se numa importante opção de controle de plantas daninhas em cerca de 65 culturas em todo o mundo, inclusive na cultura do arroz, e no controle de plantas arbustivas ou tóxicas (Burnside et al., 1996; Rodrigues \& Almeida, 1998).

Neste longo período de uso comercial do herbicida 2,4-D e de outros compostos pertencentes ao mesmo grupo químico, conhecido como reguladores de crescimento ou mimetizadores da auxina, 20 espécies de plantas daninhas tem desenvolvido resistência, sendo que 12 ecótipos apresentam resistência para o herbicida 2,4-D (Heap, 2001). Das espécies identificadas como resistentes, três são de espécies infestantes de áreas de arroz irrigado: Fimbristylis miliacea, Limnocharis flava e Sphenoclea zeylanica (Heap, 2001).

As lavouras de arroz irrigado caracterizam-se geralmente pela ocorrência de uma grande diversidade de espécies de plantas daninhas, incluindo gramíneas, ciperáceas e folhas largas, algumas das quais com hábitos aquáticos. Devido ao baixo custo de controle, os herbicidas à base de 2,4-D foram por muito tempo utilizados em aplicações seqüenciais ou em mistura de tanque com herbicidas com ação predominante sobre gramíneas, como é o caso dos produtos à base de propanil, para melhorar o espectro da aplicação. As recomendações mais freqüentes para uso de 2,4-D e outros herbicidas mimetizadores de auxina em arroz referem-se a aplicações tardias, efetuadas para o controle de plantas daninhas remanescentes de controle deficiente, efetuado na época de aplicação normal ou resultantes de reinfestação (Smith \& Shaw, 1966; Louisiana ..., 1999).

As doses recomendadas no rótulo, 200 a $400 \mathrm{~g} / \mathrm{ha} \mathrm{e.a.}$ da formulação éster e 200 a $1100 \mathrm{~g} / \mathrm{ha}$ e.a. da formulação amina (Rodrigues \& Almeida, 1998; Andrei, 1999), dos herbicidas à base de 2,4-D, registrados para uso em arroz irrigado, podem ser consideradas altas devido à elevada fitotoxicidade que podem causar ao arroz (Noldin, 1999; Cobucci \& Portela, 2000; Noldin \& Eberhardt, 2000). A seletividade do herbicida 2,4-D à cultura do arroz é reduzida quando aplicado na fase de perfilhamento ou durante a fase reprodutiva (Cobucci \& Noldin, 1999).

Além da maior sensibilidade do arroz ao herbicida 2,4-D em algumas fases de desenvolvimento da planta, fato- res como toxicidade do produto, risco de deriva para culturas sensíveis, especialmente em área onde é efetuada aplicação aérea, também tem restringido a utilização de herbicidas à base de 2,4-D na cultura do arroz irrigado. Constata-se que todos os produtos comerciais à base de 2,4-D que estão no mercado e são utilizáveis na cultura do arroz, pertencem às classes toxicológicas I e II, consideradas as mais tóxicas (Fleck, 2000).

O uso de herbicidas do grupo dos mimetizadores de auxina em arroz irrigado reduziu muito nos últimos 10 anos, principalmente após o registro no Brasil de vários herbicidas inibidores da enzima ALS (acetolactato sintase), especialmente do grupo químico das sulfoniluréias, que apresentam boa eficiência sobre várias plantas daninhas, mono e dicotiledôneas. No entanto, após a constatação da ocorrência de ecótipos de plantas daninhas resistentes aos herbicidas inibidores da ALS, especialmente aqueles do grupo químico das sulfoniluréias (Noldin et al., 1999 e 2000), os herbicidas à base de 2,4-D poderão novamente constituir-se numa opção de controle em áreas de arroz irrigado.

Assim sendo, faz-se necessário a avaliação de doses mais baixas visando a atualização das recomendações. Este trabalho, juntamente com outro similar conduzido na Estação Experimental do Arroz/IRGA, no Rio Grande do Sul, foi conduzido após sugestões da subcomissão de manejo de plantas daninhas, integrante da Comissão Técnica de Arroz da região I - CTAR I. O objetivo deste estudo foi avaliar a eficácia e seletividade do herbicida 2,4-D, aplicado em diferentes doses, épocas e formulações em arroz irrigado.

\section{MATERIAL E MÉTODOS}

Um experimento foi conduzido em campo, em duas estações de crescimento, na Estação Experimental de Itajaí/ Epagri, nas safras 1996/97 e 1997/98, para avaliar a eficácia e seletividade de quatro doses, duas épocas de aplicação e duas formulações (amina e éster) do herbicida 2,4-D em arroz irrigado.

O delineamento experimental utilizado foi o de blocos casualizados, com quatro repetições, em parcelas de $3 \mathrm{~m}$ x $5 \mathrm{~m}$. A aspersão dos herbicidas foi realizada numa faixa de $2 \mathrm{~m} \times 5 \mathrm{~m}$. As doses da formulação éster foram 56, 112, 224 e 336 g/ha e.a., na safra 1996/97, e de 100, 200, 400 e $600 \mathrm{~g} /$ ha e.a., na safra 1997/98. Para a formulação amina, as doses avaliadas foram 200, 400, 800 e $1200 \mathrm{~g} / \mathrm{ha}$ e.a., nas duas safras (Tabelas 1 e 2). Os herbicidas foram aspergidos com pulverizador costal de pressão constante $\left(\mathrm{CO}_{2}\right)$, equipado com 


\section{2,4-D em arroz irrigado}

Tabela 1. Efeito de formulação, doses e épocas de aplicação de 2,4-D na fitotoxicidade, controle das plantas daninhas Heteranthera reniformis (HETRE), Fimbristylis miliacea (FIMMI), Ludwigia longifolia (LUDLO) e L. octovalis (LUDOC), estatura de plantas, esterilidade de espiguetas e produtividade de grãos de arroz irrigado. Epagri, Itajaí, SC. 1996/97.

\begin{tabular}{|c|c|c|c|c|c|c|c|c|c|}
\hline \multirow{2}{*}{ Tratamento $^{1}$} & \multirow{2}{*}{$\begin{array}{c}\text { Dose } \\
\text { (g/ha i.a. } \\
\text { ou e.a.) }\end{array}$} & \multirow{2}{*}{$\begin{array}{c}\text { Fitotoxi- } \\
\text { cidade } \\
(\%)^{4}\end{array}$} & \multicolumn{4}{|c|}{ Controle (\%) } & \multirow{2}{*}{$\begin{array}{l}\text { Estatura } \\
\text { de } \\
\text { plantas } \\
\text { (cm) }\end{array}$} & \multirow{2}{*}{$\begin{array}{c}\text { Esterili- } \\
\text { dade } \\
(\%)\end{array}$} & \multirow{2}{*}{$\begin{array}{c}\text { Produti } \\
\text { vidade } \\
\text { (t/ha) }\end{array}$} \\
\hline & & & HETRE $^{5}$ & FIMMI $^{6}$ & LUDLO $^{6}$ & LUDOC $^{6}$ & & & \\
\hline 2,4-D éster ${ }^{2}$ & 56 & 0 & $27 \mathrm{ef}^{7}$ & $35 \mathrm{e}$ & $71 a b c$ & $72 \mathrm{ab}$ & $95 \mathrm{a}$ & $12 \mathrm{~b}$ & 3,94 bcd \\
\hline 2,4-D éster ${ }^{2}$ & 112 & 2 & $37 \mathrm{de}$ & $55 \mathrm{~d}$ & $86 \mathrm{a}$ & $86 a$ & $98 \mathrm{a}$ & $14 \mathrm{ab}$ & $6,84 a b c$ \\
\hline 2,4-D éster ${ }^{2}$ & 224 & 2 & $77 \mathrm{abc}$ & $82 a b c$ & $91 \mathrm{a}$ & $91 \mathrm{a}$ & $91 \mathrm{a}$ & $15 \mathrm{ab}$ & $7,04 \mathrm{ab}$ \\
\hline 2,4-D éster ${ }^{2}$ & 336 & 10 & $89 a b$ & $92 \mathrm{a}$ & 94 a & $94 \mathrm{a}$ & $99 \mathrm{a}$ & $13 \mathrm{~b}$ & $6,37 \mathrm{abc}$ \\
\hline 2,4-D amina ${ }^{2}$ & 200 & 5 & $65 \mathrm{bcd}$ & $84 a b$ & $92 \mathrm{a}$ & $92 \mathrm{a}$ & $95 \mathrm{a}$ & $14 a b$ & 4,16 bcd \\
\hline $2,4-\mathrm{D} \operatorname{amina}^{2}$ & 400 & 16 & $87 \mathrm{ab}$ & $94 \mathrm{a}$ & $95 \mathrm{a}$ & $95 \mathrm{a}$ & $98 \mathrm{a}$ & $18 \mathrm{ab}$ & $4,57 \mathrm{a}-\mathrm{d}$ \\
\hline 2,4-D amina ${ }^{2}$ & 800 & 37 & $94 \mathrm{ab}$ & $95 \mathrm{a}$ & $95 \mathrm{a}$ & $95 \mathrm{a}$ & $94 \mathrm{a}$ & $13 \mathrm{~b}$ & $6,49 a b c$ \\
\hline 2,4-D amina ${ }^{2}$ & 1200 & 62 & $100 \mathrm{a}$ & $92 \mathrm{a}$ & $94 \mathrm{a}$ & $94 \mathrm{a}$ & 99 a & $17 \mathrm{ab}$ & 5,17 a-d \\
\hline 2,4-D éster ${ }^{3}$ & 56 & 0 & $22 \mathrm{ef}$ & $25 \mathrm{e}$ & $30 \mathrm{e}$ & $30 \mathrm{c}$ & $97 \mathrm{a}$ & $15 \mathrm{ab}$ & $4,09 \mathrm{bcd}$ \\
\hline 2,4-D éster ${ }^{3}$ & 112 & 0 & 27 ef & $61 \mathrm{~cd}$ & $42 \mathrm{de}$ & $42 c$ & $99 \mathrm{a}$ & $17 \mathrm{ab}$ & $5,62 a b c$ \\
\hline 2,4-D éster ${ }^{3}$ & 224 & 0 & $75 \mathrm{abc}$ & $79 a b c$ & $76 a b$ & $76 a b$ & $91 \mathrm{a}$ & $14 \mathrm{~b}$ & 4,86 a-d \\
\hline 2,4-D éster ${ }^{3}$ & 336 & 12 & $84 a b$ & $87 \mathrm{a}$ & $70 a b c$ & $77 \mathrm{ab}$ & $92 \mathrm{a}$ & $14 a b$ & $4,74 \mathrm{a}-\mathrm{d}$ \\
\hline 2,4-D amina ${ }^{3}$ & 200 & 0 & 30 ef & $76 a b c$ & $55 \mathrm{~b}-\mathrm{e}$ & $55 \mathrm{bc}$ & $89 \mathrm{a}$ & $13 \mathrm{~b}$ & $2,13 \mathrm{~d}$ \\
\hline 2,4-D amina ${ }^{3}$ & 400 & 0 & 47 cde & $91 \mathrm{a}$ & $69 a-d$ & $74 a b$ & $92 \mathrm{a}$ & $12 \mathrm{~b}$ & $6,72 a b c$ \\
\hline 2,4-D amina ${ }^{3}$ & 800 & 0 & $86 a b$ & 94 a & $90 \mathrm{a}$ & $92 \mathrm{a}$ & $89 a$ & $15 a b$ & 5,18 a-d \\
\hline 2,4-D amina ${ }^{3}$ & 1200 & 0 & $91 \mathrm{ab}$ & $94 \mathrm{a}$ & $94 \mathrm{a}$ & $94 \mathrm{a}$ & $92 \mathrm{a}$ & $14 \mathrm{~b}$ & $6,68 a b c$ \\
\hline Pirazosulfuron $^{2}$ & 17,5 & 0 & $77 \mathrm{abc}$ & $91 \mathrm{a}$ & $86 a$ & $86 a$ & $91 \mathrm{a}$ & $15 \mathrm{~b}$ & $6,84 a b c$ \\
\hline Pirazosulfuron $^{3}$ & 17,5 & 0 & $5 \mathrm{f}$ & 65 bcd & 45 cde & $45 \mathrm{c}$ & $90 \mathrm{a}$ & $10 \mathrm{~b}$ & $5,86 a b c$ \\
\hline Propanil $^{2}$ & 2880 & 0 & $72 a b c$ & $84 \mathrm{ab}$ & $76 \mathrm{ab}$ & $76 \mathrm{ab}$ & $94 \mathrm{a}$ & $15 \mathrm{ab}$ & $6,97 \mathrm{ab}$ \\
\hline \multicolumn{10}{|l|}{ Propanil + } \\
\hline 2,4-D amina ${ }^{2}$ & $2880+200$ & 0 & $86 a b$ & $92 \mathrm{a}$ & $94 \mathrm{a}$ & $94 \mathrm{a}$ & $94 \mathrm{a}$ & $21 \mathrm{a}$ & $7,50 \mathrm{a}$ \\
\hline Testemunha & - & 0 & $0 \mathrm{f}$ & $0 \mathrm{f}$ & $0 \mathrm{f}$ & $0 \mathrm{~d}$ & $93 \mathrm{a}$ & $15 a b$ & $3,67 \mathrm{~cd}$ \\
\hline C.V. $(\%)$ & - & - & 32,4 & 12,3 & 23,6 & 22,5 & 8,4 & 28,7 & 33,7 \\
\hline
\end{tabular}

12,4-D éster (U-46 D-éster 2,4-D - 400 g/l e.a.); 2,4-D amina (U-46 D-Fluid 2,4-D - 720 g/l e.a.); Pirazosulfuron (Sirius SC - 250 g/l i.a.) e Propanil (Grassaid - $360 \mathrm{~g} / \mathrm{l}$ i.a.); ${ }^{2}$ Aplicação em pós-emergência normal; ${ }^{3}$ Aplicação em pós-emergência tardia; ${ }^{4}$ Avaliação aos 16 DAA (dias após a aplicação dos tratamentos); ${ }^{5}$ Avaliações aos $16 \mathrm{DAA} ;{ }^{6} \mathrm{Avaliação} \mathrm{em} \mathrm{pré-colheita;}{ }^{7}$ Médias seguidas da mesma letra, na coluna, não diferem significativamente entre si (Duncan 5\%).

quatro bicos Teejet série 110.02 , espaçados de $50 \mathrm{~cm}$ entre si e resultando em consumo de calda de 200 1/ha. A aplicação foi realizada em duas épocas: época 1 (E1), herbicidas aplicados em pós-emergência, com arroz no estádio de 3-4 folhas, e época 2 (E2), herbicidas aplicados em pós-emergência tardia. Na safra 1996/97, as aspersões dos herbicidas foram realizadas aos 16 e 38 dias após a semeadura e na safra 1997/98, foram realizadas aos 17 e 39 dias após a semeadura, respectivamente, nas épocas E1 e E2.

As práticas de preparo do solo, adubação, semeadura e controle de pragas, foram executadas de acordo com as recomendações do sistema de produção de arroz irrigado no sistema pré-germinado em Santa Catarina (Epagri, 1998). As semeaduras do arroz foram realizadas nos dias 11/11/96 e 21/11/97, na densidade de $120 \mathrm{~kg} / \mathrm{ha}$. A cultivar utilizada como reagente foi a Epagri 108, de ciclo longo (média de 145-150 dias da semeadura à maturação na região de Itajaí).

As plantas daninhas avaliadas no experimento foram: aguapé (Heteranthera reniformis $=$ HETRE), cruz-de-malta (Ludwigia longifolia $=$ LUDLO e L. octovalvis $=$ LUDOC) e cuminho (Fimbristylis miliacea = FIMMI). Além destas, verificou-se a presença de capim-arroz (Echinochloa crusgalli e E. colona) em toda a área experimental. Na safra 1997/98, toda a área experimental recebeu uma aplicação do produto comercial Furore, $120 \mathrm{~g} / \mathrm{l}$ de fenoxaprop-ethyl, na dose de $60 \mathrm{~g} / \mathrm{ha}$ i.a., aos 40 dias após a semeadura.

A eficácia dos herbicidas sobre as plantas daninhas e a fitotoxicidade no arroz foram avaliadas através de observações visuais, seguindo uma escala de zero a 100 , onde zero corresponde a nenhum controle ou fitotoxicidade e 100 
Tabela 2. Efeito de formulação, doses e épocas de aplicação de 2,4-D na fitotoxicidade, controle das plantas daninhas Heteranthera reniformis (HETRE), Fimbristylis miliacea (FIMMI), Ludwigia longifolia (LUDLO) e L. octovalis (LUDOC), estatura de plantas, esterilidade de espiguetas e produtividade de grãos de arroz irrigado. Epagri, Itajaí, SC. 1997/98.

\begin{tabular}{|c|c|c|c|c|c|c|c|c|c|}
\hline \multirow[b]{2}{*}{ Tratamento ${ }^{1}$} & \multirow{2}{*}{$\begin{array}{c}\text { Dose } \\
\text { (g/ha i.a. } \\
\text { ou e.a.) }\end{array}$} & \multirow{2}{*}{$\begin{array}{c}\text { Fitotoxi- } \\
\text { cidade } \\
(\%)^{4}\end{array}$} & \multicolumn{4}{|c|}{ Controle $(\%)$} & \multirow{2}{*}{$\begin{array}{l}\text { Estatura } \\
\text { de } \\
\text { plantas } \\
(\mathrm{cm})\end{array}$} & \multirow{2}{*}{$\begin{array}{c}\text { Esteri- } \\
\text { lidade } \\
(\%)\end{array}$} & \multirow{2}{*}{$\begin{array}{c}\text { Produti } \\
\text { vidade } \\
\text { (t/ha) }\end{array}$} \\
\hline & & & HETRE $^{5}$ & FIMMI $^{6}$ & LUDLO $^{6}$ & LUDOC $^{6}$ & & & \\
\hline 2,4-D éster ${ }^{2}$ & 100 & 0 & $60 b^{7}$ & $100 \mathrm{a}$ & $97 \mathrm{a}$ & $97 \mathrm{a}$ & $101 \mathrm{ab}$ & 12 bcd & $6,37 \mathrm{abc}$ \\
\hline $2,4-\mathrm{D}$ éster $^{2}$ & 200 & 1 & $74 \mathrm{ab}$ & $100 \mathrm{a}$ & $97 \mathrm{a}$ & $97 \mathrm{a}$ & $99 \mathrm{ab}$ & $11 \mathrm{~cd}$ & $6,86 \mathrm{a}$ \\
\hline 2,4-D éster ${ }^{2}$ & 400 & 1 & $100 \mathrm{a}$ & $100 \mathrm{a}$ & $100 \mathrm{a}$ & $100 \mathrm{a}$ & $103 \mathrm{ab}$ & $13 \mathrm{bcd}$ & 5,88 a-e \\
\hline 2,4-D éster ${ }^{2}$ & 600 & 5 & $94 \mathrm{ab}$ & $100 \mathrm{a}$ & $100 \mathrm{a}$ & $100 \mathrm{a}$ & $103 \mathrm{ab}$ & 13 bcd & $6,48 \mathrm{ab}$ \\
\hline $2,4-\mathrm{D}$ amina $^{2}$ & 200 & 0 & $73 \mathrm{ab}$ & $100 \mathrm{a}$ & $100 \mathrm{a}$ & $100 \mathrm{a}$ & $102 \mathrm{ab}$ & 13 bcd & 4,97 b-e \\
\hline $2,4-\mathrm{D}$ amina $^{2}$ & 400 & 0 & $100 \mathrm{a}$ & $100 \mathrm{a}$ & $100 \mathrm{a}$ & $100 \mathrm{a}$ & $101 \mathrm{ab}$ & $12 \mathrm{~cd}$ & $6,06 \mathrm{a}-\mathrm{d}$ \\
\hline $2,4-\mathrm{D}$ amina $^{2}$ & 800 & 0 & $100 \mathrm{a}$ & $100 \mathrm{a}$ & $100 \mathrm{a}$ & $100 \mathrm{a}$ & $99 \mathrm{ab}$ & 13 bcd & 5,39 a-e \\
\hline $2,4-\mathrm{D}$ amina $^{2}$ & 1200 & 8 & $100 \mathrm{a}$ & $100 \mathrm{a}$ & $100 \mathrm{a}$ & $100 \mathrm{a}$ & $102 \mathrm{ab}$ & $15 \mathrm{a}-\mathrm{d}$ & 5,41 a-e \\
\hline 2,4-D éster ${ }^{3}$ & 100 & 10 & $100 \mathrm{a}$ & $85 \mathrm{~b}$ & $74 \mathrm{c}$ & $74 \mathrm{c}$ & $102 \mathrm{ab}$ & $19 a$ & $4,44 \mathrm{de}$ \\
\hline 2,4-D éster ${ }^{3}$ & 200 & 10 & $94 \mathrm{ab}$ & $100 \mathrm{a}$ & $82 a b c$ & $82 a b c$ & $99 \mathrm{ab}$ & $17 \mathrm{ab}$ & 5,63 a-e \\
\hline 2,4-D éster ${ }^{3}$ & 400 & 19 & $100 \mathrm{a}$ & $100 \mathrm{a}$ & $91 \mathrm{ab}$ & $91 \mathrm{ab}$ & $101 \mathrm{ab}$ & $16 a b c$ & 5,07 a-e \\
\hline 2,4-D éster ${ }^{3}$ & 600 & 20 & $97 \mathrm{a}$ & $100 \mathrm{a}$ & $100 \mathrm{a}$ & $100 \mathrm{a}$ & $97 \mathrm{~b}$ & 15 a-d & $4,14 \mathrm{e}$ \\
\hline 2,4-D amina $^{3}$ & 200 & 5 & $94 \mathrm{ab}$ & $100 \mathrm{a}$ & $77 \mathrm{bc}$ & $77 \mathrm{bc}$ & $102 \mathrm{ab}$ & $16 a b c$ & 4,64 cde \\
\hline $2,4-\mathrm{D}$ amina $^{3}$ & 400 & 14 & $93 \mathrm{ab}$ & $100 \mathrm{a}$ & $82 a b c$ & $82 \mathrm{abc}$ & $102 \mathrm{ab}$ & $14 \mathrm{bcd}$ & $4,68 \mathrm{~b}-\mathrm{e}$ \\
\hline $2,4-\mathrm{D}$ amina $^{3}$ & 800 & 15 & $100 \mathrm{a}$ & $100 \mathrm{a}$ & $100 \mathrm{a}$ & $100 \mathrm{a}$ & $104 \mathrm{a}$ & 15 a-d & $4,24 \mathrm{de}$ \\
\hline $2,4-\mathrm{D}$ amina $^{3}$ & 1200 & 16 & $100 \mathrm{a}$ & $100 \mathrm{a}$ & $97 \mathrm{a}$ & $97 \mathrm{a}$ & $99 \mathrm{ab}$ & $15 a-d$ & $4,07 \mathrm{e}$ \\
\hline Pirazosulfuron $^{2}$ & 17,5 & 1 & $95 \mathrm{ab}$ & $100 \mathrm{a}$ & $100 \mathrm{a}$ & $100 \mathrm{a}$ & $100 \mathrm{ab}$ & $13 \mathrm{bcd}$ & 5,56 a-e \\
\hline Pirazosulfuron $^{3}$ & 17,5 & 0 & $74 \mathrm{ab}$ & $100 \mathrm{a}$ & $85 a b c$ & $85 a b c$ & $103 \mathrm{ab}$ & $10 \mathrm{~d}$ & 5,24 a-e \\
\hline Propanil $^{2}$ & 2880 & 0 & $91 \mathrm{ab}$ & $100 \mathrm{a}$ & $100 \mathrm{a}$ & $100 \mathrm{a}$ & $102 a b$ & $11 \mathrm{~cd}$ & $6,40 a b c$ \\
\hline \multicolumn{10}{|l|}{ Propanil + } \\
\hline 2,4-D amina ${ }^{2}$ & $2880+200$ & 4 & $97 \mathrm{a}$ & $100 \mathrm{a}$ & $100 \mathrm{a}$ & $100 \mathrm{a}$ & $100 \mathrm{ab}$ & $12 \mathrm{~cd}$ & $6,84 \mathrm{a}$ \\
\hline Testemunha & - & 0 & $0 \mathrm{c}$ & $0 \mathrm{c}$ & $0 \mathrm{~d}$ & $0 \mathrm{~d}$ & $102 a b$ & $13 \mathrm{bcd}$ & 5,27 a-e \\
\hline C.V. (\%) & - & - & 25,5 & 5,5 & 11,7 & 11,7 & 3,5 & 22,2 & 19,8 \\
\hline
\end{tabular}

12,4-D éster (U-46 D-éster 2,4-D - 400 g/l e.a.); 2,4-D amina (U-46 D-Fluid 2,4-D - 720 g/l e.a.); Pirazosulfuron (Sirius SC - 250 g/l i.a.) e Propanil (Grassaid - 360 g/l i.a.); ${ }^{2}$ Aplicação em pós-emergência normal; ${ }^{3}$ Aplicação em pós-emergência tardia; ${ }^{4}$ Avaliação aos 15 DAA (dias após a aplicação dos tratamentos); ${ }^{5}$ Avaliações aos $40 \mathrm{DAA} ;{ }^{6}$ Avaliação em pré-colheita; ${ }^{7}$ Médias seguidas da mesma letra, na coluna, não diferem significativamente entre si (Duncan 5\%).

corresponde ao controle total das plantas daninhas ou morte das plantas de arroz.

A produtividade de grãos do arroz foi avaliada pela colheita das panículas de arroz em uma área de $1,5 \mathrm{~m}$ x 3,0 m $\left(4,5 \mathrm{~m}^{2}\right)$ em cada parcela e o peso corrigido para $13 \%$ de umidade.

\section{RESULTADOS E DISCUSSÃO}

Os resultados das avaliações efetuadas nas safras 1996/ 97 e 1997/98, constam, respectivamente, nas Tabelas 1 e 2.

Na safra 1996/97, os tratamentos com 2,4-D na formulação éster (336 g/ha), aplicados nas duas épocas, e na formulação amina (400, 800 e 1200 g/ha), aplicados na época normal (E1), causaram sintomas típicos de fitotoxicidade como enrolamento das folhas ("acebolamento") e emissão de raízes adventícias na base das plantas, aos 16 DAA (dias após a aplicação) (Tabela 1). Os danos mais severos foram observados com os tratamentos 2,4-D amina nas doses de 800 e $1200 \mathrm{~g} / \mathrm{ha}$, com injúria média de 37 e $62 \%$, respectivamente. Na safra 1997/98, os sintomas mais severos de fitotoxicidade foram observados nos tratamentos com as formulações éster e amina, em todas as doses aplicadas na E2 e nas doses mais elevadas da E1 (Tabela 2). Apesar das plantas de arroz terem apresentado recuperação no decorrer do ciclo e apenas sintomas leves de fitotoxicidade foram observados visualmente na avaliação final efetuada antecedendo a colheita, ocorreu redução na produtividade do arroz nos trata- 
mentos com 2,4-D nas doses de 600 e $1200 \mathrm{~g} / \mathrm{ha}$, respectivamente para as formulações éster e amina aplicados na E2 (Tabela 2).

Os tratamentos 2,4-D amina nas doses de 800 e 1200 $\mathrm{g} /$ ha aplicados na E1 e $1200 \mathrm{~g} / \mathrm{ha}$ aplicado na E2, na safra de 1996/97, proporcionaram mais de 90\% de controle de aguapé (HETRE). Na segunda safra (1997/98), a aplicação de 2,4-D éster nas doses de 400 e $600 \mathrm{~g} / \mathrm{ha}$ aplicado na E1 e nas doses de 100, 200, 400 e $600 \mathrm{~g} / \mathrm{ha}$ na E2, controlou mais de $90 \%$ a espécie HETRE (Tabela 2). Para a mesma espécie, 2,4-D amina aplicado nas doses de 400, 800 e $1200 \mathrm{~g} / \mathrm{ha}$ na E1 e nas doses de 200, 400, 800 e 1200 g/ha na E2, controlou mais de $90 \%$. Pirazosulfuron, propanil isoladamente e em mistura com 2,4-D amina, controlaram adequadamente HETRE somente quando aplicado na El no segundo ano de avaliação (Tabelas 1 e 2).

Na safra 1996/97, os tratamentos 2,4-D éster (336 g/ ha), 2,4-D amina (400, 800 e $1200 \mathrm{~g} / \mathrm{ha})$, pirazosulfuron e propanil + 2,4-D controlaram mais de $90 \%$ do cuminho (FIMMI) na E1. Na aplicação tardia, os tratamentos que proporcionaram mais de $90 \%$ de controle de cuminho foram 2,4-D amina nas doses de 400, 800 e $1200 \mathrm{~g} /$ ha (Tabela 1). Na safra 1997/98, todos os tratamentos, exceto 2,4-D éster ( $100 \mathrm{~g} / \mathrm{ha}$ ) na época E2, controlaram $100 \%$ a espécie FIMMI (Tabela 2).

Em ambas as safras, as duas espécies de cruz-de-malta (LUDLO e LUDOC) presentes no experimento, mostraram-se bastante suscetíveis aos tratamentos herbicidas quando aplicados no estádio inicial de desenvolvimento (E1). Na safra 96/97, os tratamentos com 2,4-D nas duas formulações (éster e amina), exceto as doses mais baixas de 2,4-D éster (56 e $112 \mathrm{~g} / \mathrm{ha}$ ) e a mistura de propanil mais 2,4-D amina, aplicados na E1, apresentaram bom $(85-90 \%)$ a excelente ( $>90 \%$ ) controle de cruz-de-malta. Nas duas safras, apenas as doses mais elevadas de 2,4-D amina ( 800 e $1200 \mathrm{~g} / \mathrm{ha}$ ) em aplicação tardia e éster (400 e 600 g/ha) na safra 1997/98, resultaram em controle adequado (superior a 90\%) de LUDLO e LUDOC.

Na safra 1996/97, não foi observado nenhum efeito significativo dos tratamentos herbicidas sobre a estatura das plantas de arroz (Tabela 1). Na segunda safra (1997/98), a aplicação de $400 \mathrm{~g} / \mathrm{ha}$ na E2 de 2,4-D na formulação éster reduziu a estatura das plantas, comparado com a aplicação de $800 \mathrm{~g} / \mathrm{ha}$ de 2,4-D na formulação amina na mesma época (Tabela 2). No entanto, a estatura de plantas em nenhum destes tratamentos diferenciou-se dos demais, incluindo a testemunha sem a aplicação de herbicidas.

Não foi observado efeito significativo dos tratamentos sobre a esterilidade de espiguetas, comparado com o tratamento testemunha, mesmo nos tratamentos com aplicação tardia de doses elevadas de 2,4-D, na safra 1996/97 (Tabela 1). No segundo ano (1997/98), apenas o tratamento com 2,4D amina (1200 g/ha) na E1 apresentou esterilidade de grãos significativamente superior à observada na testemunha (Tabela 2). O fato de não ter sido detectado nestes experimentos nenhum efeito significativo da aplicação tardia de 2,4-D no aumento da esterilidade de espiguetas, pode ser devido à época de aplicação (E2) ter sido efetuada pelo menos 30 dias antes da diferenciação do primórdio floral. A aplicação foi efetuada aos 38 e 39 dias após a semeadura do arroz, em 1996/97 e 1997/98, respectivamente, mas a cultivar utilizada, Epagri 108, possui ciclo longo e o início do desenvolvimento da panícula ocorre 70-80 dias após a semeadura. Menezes et al. (1997), em trabalho conduzido na Estação Experimental do Arroz do IRGA, também não observaram efeito significativo do herbicida 2,4-D sobre o número de panículas $/ \mathrm{m}^{2}$, número de espiguetas por panícula e na esterilidade de espiguetas do arroz, independente da dose ou época de aplicação.

$\mathrm{Na}$ safra 1996/97, os tratamentos propanil + 2,4-D amina, propanil isoladamente e 2,4-D éster (112 g/ha), aplicados na E1, resultaram em produtividade de grãos significativamente superiores à testemunha sem controle (Tabela 1). No segundo ano, a produtividade de grãos de arroz variou significativamente entre os tratamentos avaliados, mas estes não se diferenciaram significativamente da testemunha sem controle (Tabela 2). As piores produtividades foram obtidas nas doses mais elevadas de 2,4-D, em ambas as formulações.

De maneira geral, observou-se que tanto a aplicação de 2,4-D na formulação éster como na formulação amina, aplicados nas doses mais baixas ( $100 \mathrm{~g} / \mathrm{ha}$ da formulação éster e $200 \mathrm{~g} / \mathrm{ha}$ da formulação amina) e na E1, proporcionaram controle adequado das plantas daninhas FIMMI, LUDLO e LUDOC. A espécie HETRE mostrou-se mais tolerante a este herbicida, necessitando doses mais elevadas para o controle adequado da mesma. Confirmou-se ainda neste trabalho de pesquisa, resultados já relatados por outros pesquisadores, que na aplicação mais precoce dos herbicidas, pode-se utilizar doses mais baixas e obter controle eficiente das plantas daninhas.

\section{CONCLUSÕES}

Em áreas com grande número de espécies daninhas (folhas largas, ciperáceas e gramíneas), é recomendável a aplicação associada de herbicidas com diferentes espectros de ação para a obtenção de níveis de controle mais adequados e que previnam as perdas na produtividade do arroz. A aplicação de 2,4-D nas formulações amina ou éster deve ser preferencialmente efetuada antes do perfilhamento do arroz. Aplicações mais precoces destes herbicidas permitem o uso de doses mais baixas com melhor controle das plantas daninhas e menor fitotoxicidade ao arroz. No entanto, é importante ressaltar que para a aplicação em pós-emergência na época normal (até 20 dias após a semeadura), existem diversas opções no mercado de herbicidas que apresentam menores riscos ambientais e mais seletivos ao arroz que aqueles à base de 2,4-D. Em áreas de ocorrência de resistência à herbicidas destas espécies estudadas, herbicidas a base de 2,4$\mathrm{D}$ podem se constituir em alternativa de controle. 


\section{AGRADECIMENTOS}

O autor agradece aos assessores científicos pela revisão e sugestões apresentadas para o aprimoramento deste trabalho; ao Eng. Agr., M.Sc., Domingos Sávio Eberhardt, pela colaboração e sugestões, bem como ao Técnico Agrícola Estevão Tirelli, pelo apoio dado nas atividades de campo.

\section{LITERATURA CITADA}

ANDREI, E. Compêndio de defensivos agrícolas. São Paulo: Andrei, 1999. 672p.

BURNSIDE, O.C. et al. Biologic and economic assessment of benefits from use of phenoxy herbicides in the United States. Washington, DC: National Agricultural Pesticide Impact Assessment Program/USDA/CSREES, 1996. np. (NAPIAP Report Number 1-PA-96).

COBUCCI, T.; NOLDIN, J.A. Plantas daninhas e seu controle. In: VIEIRA, N.R.; SANTOS, A.B. dos; SANT'ANA, E.P., ed. A cultura do arroz no Brasil. Santo Antonio de Goiás: Embrapa Arroz e Feijão, 1999. p.375-415.

COBUCCI, T.; PORTELA, C.M.O. Seletividade de herbicidas aplicados em diferentes estádios de crescimento do arroz em terras altas. In: CONGRESSO BRASILEIRO DA CIÊNCIA DAS PLANTAS DANINHAS, 22. Foz do Iguaçu, 2000 Resumos... Londrina: SBCPD, 2000. p.217.

EPAGRI. Sistema de produção de arroz irrigado em Santa Catarina: (pré-germinado). Florianópolis: Epagri, 1998. 79p. (Epagri. Sistema de Produção, 32).

FLECK, N.G. Controle de plantas daninhas na cultura do arroz irrigado através da aplicação de herbicidas com ação seletiva. Porto Alegre: Ed. Autor, 2000. 32p.

HEAP, I. The international survey of herbicide resistant weeds. Disponível em: <http:// www.weedscience.com Consultado em 14 março de 2001.
LOUISIANA rice production handbook. Batton Rouge: Louisiana State University Agricultural Center, 1999. 116p.

MENEZES, V.G.; ANDRES, A.; FLECK, N.G. Controle de angiquinho (Aeschynomene denticulata) em arroz irrigado através do herbicida 2,4-D nas formulações amina e éster em dois estágios de desenvolvimento da cultura. In: REUNIÃO DA CULTURA DO ARROZ IRRIGADO, 22. Balneário Camboriú, 1997. Anais... Itajaí: Epagri, 1997. p.356-359.

NOLDIN, J.A. Doses, épocas e formulações de 2,4-D em arroz irrigado. In: CONGRESSO BRASILEIRO DE ARROZ IRRIGADO, 1 e REUNIÃO DA CULTURA DO ARROZ IRRIGADO, 23. Pelotas, 1999. Anais... Pelotas: Embrapa Clima Temperado, 1999. p.562-565.

NOLDIN, J.A.; EBERHARDT, D.S; KNOBLAUCH, R. Resistência de Sagittaria montevidensis a herbicidas: primeiras evidências. In: CONGRESSO BRASILEIRO DE ARROZ IRRIGADO, 1 e REUNIÃO DA CULTURA DO ARROZ IRRIGADO, 23. Pelotas, 1999. Anais... Pelotas: Embrapa Clima Temperado, 1999. p.566-569.

NOLDIN, J.A.; EBERHARDT, D.S. Controle de sagitária resistente a herbicidas com aplicações em pulverização. In: CONGRESSO BRASILEIRO DA CIÊNCIA DAS PLANTAS DANINHAS, 22. Foz do Iguaçu, 2000. Resumos... Londrina: SBCPD, 2000. p.515.

NOLDIN, J.A.; EBERHARDT, D.S.; CHEHADE, A.T.; DITTRICH, R.C. Sagitária resistente a herbicidas inibidores da ALS In: CONGRESSO BRASILEIRO DA CIÊNCIA DAS PLANTAS DANINHAS, 22. Foz do Iguaçu, 2000. Resumos... Londrina: SBCPD, 2000. p.514.

RODRIGUES, B.N.; ALMEIDA, F.S. de. Guia de herbicidas. Londrina: Edição dos Autores, 1998. 648 p.

SMITH, R.J.; SHAW, W.C. Weeds and their control in rice production. Washington, DC: Agricultural Research Service/United States Department of Agriculture, 1966. $64 p$. 